\title{
Zur Theorie des Verseifungsprozesses
}

ron

R. Fanto.

Aus dem chemischen Laboratorium der $k$. $k$. Hochschule für Bodenkultur in Wien.

(Vorgelegt in der Sitzung am 9. Juni 1904.)

Die Verseifung von Fetten durch Alkalien war schon mehrfach Gegenstand eingehender Untersuchungen, ohne daß in dieser Frage eine endgültige Entscheidung zu erzielen gewesen wäre. Der dabei stattfindende Vorgang wird, und dies ist die ältere, ziemlich allgemeine Anschauung, als quadrimolekulare Reaktion aufgefaßt, indem auf ein Molekül Triglyzerid gleichzeitig 3 Moleküle $\mathrm{KOH}$ einwirken. Geite $1^{1}$ hält diese Auffassung für unrichtig und hat zu beweisen versucht, daß die Reaktion nicht quadrimolekular, sondern stufenweise verläuft, d. h. sich aus drei aufeinanderfolgenden bimolekularen Reaktionen zusammensetzt, deren Geschwindigkeitskonstanten nicht gleich sind, sondern, $K_{1}>K_{2}>K_{3}$, sich annähernd wie $3: 2: 1$ verhalten.

Der von ihm zur Beweisführung eingeschlagene Weg, die messende Verfolgung des Reaktionsverlaufes in homogener Lösung, kann aber auf den vorliegenden Fall nur dann angewendet werden, wenn es gelingt, ein Lösungsmittel ausfindig zu machen, das sowohl Fett als auch Alkali und Verseifungsprodukte in ausreichender Menge zu lösen vermag, ohne sich selbst an der Reaktion zu beteiligen. Geitel hat nun

1 Journal für prakt. Chemie, 1897 (55), 429. 
seine Versuche mit äthylalkoholischer Kalilauge ausgeführt. Ein Umstand, den er allerdings nicht berücksichtigen konnte, da derselbe erst ein Jahr nach seiner Publikation zur allgemeinen Kenntnis gelangte, machte die Beweiskraft der Geitel'schen Versuche vollkommen zu nichte.

Henriques ${ }^{1}$ hat nachgewiesen, daß sowohl in der Kälte als in der Wärme bei Anwesenheit geringer Mengen von Alkali die einsäurigen Alkohole das dreisäurige Glyzerin schon nach ganz kurzer Einwirkungsdauer (einige Minuten). vollständig oder zum allergrößten Teile unter Bildung der betreffenden Fettsäurealkylester verdrängen. Bei Besprechung der experimentellen Versuche Geitels, die er Scheinbeweise nennt, schreibt er, in richtiger Erkenntnis derselben: $\gg D a ß$ Geitel hier Äthylester, rein oder mit Glyzeriden gemischt, unter Händen hatte und daß hiefür alle seine experimentellen Angaben stimmen, ist ohneweiters ersichtlich.

Neuerdings hat nun Lewkowitsch, ${ }^{2}$ ein Anhänger der Geitel'schen Theorie, eine Reihe von Versuchen angesteilt, deren Ergebnisse er für die stufenweise Verseifung beweiskräftig hält.

Auf dieselben näher einzugehen halte ich schon deswegen für unnötig; da von Balbiano ${ }^{3}$ gezeigt wurde, daß den Versuchsresultaten von Lewkowits ch keinerlei Beweiskraft innewohne, ja daß sie zum Teile mehr zu Gunsten der älteren Theorie sprechen; weiter, daß bei der unvollständigen Verseifung von Tribenzoin mit wässeriger Kalilauge reines Tribenzoin zurückbleibt und andrerseits bei der Einwirkung von Benzoylchlorid auf überschüssiges Glyzerin wiederum reines. Tribenzoin sich bildet, also weder bei der Verseifung noch bei der Esterifizierung Zwischenprodukte, wie Di- oder Monobenzoin auftreten, Tatsachen, die mit der Theorie der stufenweisen Verseifung, wie sie von Geitel aufgefaßt wird, nicht in Einklang zu bringen sind. Aus diesen Versuchsergebnissen leitet Balbiano $a b$, daß der Verseifungsprozeß nicht sturen-

1 Zeitschrift für angew. Chemie, 1898, 700.

2 Ber. der Deutschen chem. Gesellschaft, 33, 89 (1900).

3 Gazz. chim., 32 (1), 265 (1902); Ber, der Deutschen chem. Gesellschaft,. 8,1571 (1903); 1, 155 (1904). 
weise erfolgen kann. Hiezu wäre noch zu bemerken, daß sowohl Lewkowitsch als auch Balbiano in inhomogenem System gearbeitet haben, was für den scheinbaren Verlauf der Reaktion nichts weniger als belanglos ist.

Gegen die scheinbar einfachere Annahme der direkten oder besser der quadrimolekularen Verseifung lassen sich nun aber schwerwiegende Bedenken theoretischer Natur geltend machen. Reaktionen höherer Ordnung sind nämlich sehr selten und verlaufen in der Regel nur mit ganz geringer Geschwindigkeit; der größte Teil scheinbar hochmolekularer Reaktionen hat sich in eine Reihe stufenweise sich abspielender Reaktionen niedrigerer Ordnung auflösen lassen. Es sei hier nur auf die Untersuchungen von Knoblauch ${ }^{1}$ über die Geschwindigkeit der Verseifung der Ester mehrbasischer Säuren hingewiesen. In dem von ihm untersuchten Falle, Verseifung des Diäthylbernsteinsäureesters durch Natronlauge, verläuft die Reaktion nicht, wie man anfänglich anzunehmen geneigt wäre, trimolekular, sondern sie setzt sich aus zwei Reaktionen zweiter Ordnung mit verschiedener Geschwindigkeitskonstante zusammen.

$\mathrm{Da}$ es mir bis jetzt nicht gelungen ist, ein geeignetes Lösungsmittel ausfindig zu machen, andrerseits aber die Untersuchung des Reaktionsverlaufes in inhomogener Lösung wenig aussichtsreich erschien, in Anbetracht der auf diesem Gebiete noch herrschenden Unsicherheit, mußte der Versuch einer prinzipiellen Entscheidung dieser Frage vorderhand zurïckgestellt werden.

Es ist nun ohneweiters einleuchtend, daß die Reaktion auch dann als stufenweise verlaufend charakterisiert ist, wenn es gelingt, bei Anwendung eines inhomogenen Systems die bei dieser Reaktion notwendig auftretenden Zwischenprodukte zu fassen. Die Isolierung dieser Stoffe würde aber, wie schon Geitel fand, ziemlich bedeutende experimentelle Schwierigkeiten bieten; sie ist aber gar nicht notwendig.

Denken wir uns in einem partiell verseiften Fette, nach Zersetzung der Seife durch eine geeignete Säure, einerseits die

1 Zeitschrift für physik. Chemie, 26, 96 (1898). 
Zunahme der Säurezahl bestimmt, andrerseits aber die bei der Verseifung abgespaltene Menge Glyzerin, so wird nun die gefundene Glyzerinmenge entweder der Zunabme der Säurezahl, also dem in Reaktion getretenen Kali, entsprechen, oder sie wird kleiner sein. Im ersten Falle erfolgte die Verseifung quadrimolekular, wenigstens praktisch, im zweiten stufenweise, und zwar unbedingt; im letzteren Falle wäre die Frage endgültig gelöst. Erscheint aber die Verseifung praktisch quadrimolekular, so ist dadurch der Verlauf der Reaktion noch nicht genügend aufgeklärt, da sie möglicherweise doch stufenweise erfolgen kann. Es sei z. B. die Geschwindigkeit der ersten Reaktion $K_{1}$, der zweiten $K_{2}$, der dritten $K_{3}$ gesetzt, $K_{1}<K_{2}<K_{3}$, so kann, wenn das Verhältnis der Reaktionsgeschwindigkeiten $K_{2} / K_{1} K_{3} / K_{2}$ sehr groß ausfällt, die in Wirklichkeit stufenweise verlaufende Reaktion als eine quadrimolekulare erscheinen. Die durch $R_{1}$ gebildete kleine Menge Diacylhydrin wird nach 2 sehr rasch in Monoacylhydrin und dieses noch rascher in Glyzerin und Fettsäure verwandelt, so daß in keinem Momente irgend größere analytisch bestimmbare Mengen des Di- und Monoproduktes vorhanden sind. Außer den bereits vorher besprochenen Grenzfällen gibt es also noch eine dritte Möglichkeit, die allerdings für die Praxis ganz bedeutungslos ist und nur theoretisches Interesse besitzt: »Die Reaktion verläuft genau genommen wohl stufenweise, praktisch aber quadrimolekular.« Dieser dritte Fall ist aber nach der Geitel'schen Auffassung des Reaktionsverlaufes unmöglich.

Verhalten sich nun die Reaktionsgeschwindigkeiten, wie Geitel behauptet und zu beweisen versuchte, wirklich wie $3: 2: 1$, so ist es klar, daf es unter Einhaltung gewisser Versuchsbedingungen gelingen müsse, Di- oder Monoacylhydrin zu isolieren beziehungsweise dessen Vorhandensein im partiell verseiften Fette analytisch nachzuweisen. Gelingt dies auch unter den für stufenweise Verseifung günstigsten Bedingungen nicht, so ergibt sich hieraus wohl mit einiger Sicherheit, daß entweder von einer stufenweisen Verseifung überhaupt nicht die Rede sein kann oder daß zumindest das Geitel'sche Verhältnis der Reaktionsgeschwindigkeiten im Falle 
der Fettverseifung durch Alkali nicht Platz greift. Allerdings muß nun noch berücksichtigt werden, daß die Verseifung in inhomogener Lösung schon deswegen mehr den Charakter einer quadrimolekularen annehmen wird, weil die Reaktion wohl größtenteils nur an der Berührungsfläche der Flüssigkeiten stattfindet und bei relativ kleiner Diffusionsgeschwindigkeit die Weiterveränderung etwa gebildeten Di- oder Monoacylhydrins mehr begünstigt erscheint als die Spaltung frischen Fettes.

Auch diese Betrachtung zeigt deutlich, daß eine endgültige Aufklärung des Sachverhaltes auf analytischem Wege nur dann zu erwarten ist, wenn dieser zur Annahme der stufenweisen Verseifung führt, d. h. wenn es gelingt, Anhaltspunkte für die intermediäre Bildung von Mono- und Diacylhydrin zu gewinnen; wenn dies aber, und das ist das nächste Ergebnis der vorliegenden Untersuchung, nicht der Fall ist, so kann nur gesagt werden, daß die Verseifung der Fette praktisch quadrimolekular erfolgt. $\mathrm{Zu}$ demselben Resultate ist Balbiano durch seine Versuche gekommen; er läßt nur außeracht, daß, wie oben angedeutet, der Verseifungsprozeß praktisch wohl quadrimolekular, eigentich aber doch stufenweise vor sich gehen kann. Zur Aufklärung wird es, wie ich glaube, wohl nötig sein, neuerlich auf die messende Verfolgung des Reaktionsverlaufes zurückzukommen. Dies soll geschehen, sobald ein passendes Lösungsmittel gefunden sein wird.

Meine ersten Versuche waren natürlich dahin gerichtet, in homogener Lösung zu arbeiten. Als Lösungsmittel wurde Isobutylalkohol verwendet. Dabei habe ich dieselben Erfahrungen gemacht, die Henriques (1. c.) schon vor mehreren Jahren veröffentlicht hat; ich kann nun seine Versuchsergebnisse vollinhaltlich bestätigen. Es sei nur erwähnt, daß bei einem derartigen Versuche - Leinöl wurde mit einem Drittel der von der Theorie verlangten Menge alkoholischer Kalilauge eine halbe Stunde auf $60^{\circ} \mathrm{C}$. erwärmt - einerseits das gesamte vorhandene Glyzerin abgespalten war, andrerseits im isolierten Äthylester (ohne weitere Reinigung) $95 \%$ des theoretisch vorhandenen Alkoxyls gefunden wurden. Beides, Glyzerin und Alkoxyl, wurden nach dem Jodidverfahren bestimmt. 
Die Versuche, Pyridin als Lösungsmittel zu verwenden, scheiterten, da damit ein während der Verseifung homogen bleibendes System nicht zu erzielen war. Vom Arbeiten in homogener Lösung mußte nun natürlich abgesehen werden.

Die Versuche, die mit sehr verdünnten wässerigen Kalilösungen $(n / 10$ und $n / 4)$ vorgenommen wurden, führten $z u$ keinem Resultate, da es sich herausstellte, daß derartig verdünnte Lösungen nur äußerst langsam auf Fette einwirkten. Des weiteren kam durchwegs eine wässerige Normallösung von Kalilauge zur Verwendung. Die Versuchsanordnung war folgende: Das Fett wurde in ein $25 \mathrm{~cm}$ langes, zylindrisches Gefäß von $3.5 \mathrm{~cm}$ Diam. gewogen und dasselbe nach Zusatz der Kalilösung mit einem aufrechtstehenden Rückflußkühler mit Hugershoff"scher Rührvorrichtung in Verbindung gesetzt. Der Zylinder wurde sechs respektive drei Stunden unter starkem Rühren (mittels Turbine) im siedenden Wasserbade belassen. Das Reaktionsgemisch wurde mit heißem Wasser in einen Kolben gespült, mit Äther nachgewaschen, mit Essigsäure zersetzt, abkühlen gelassen, im Scheidetrichter mit Äther ausgeschüttelt, das Wasser wiederhọlt mit Äther, der Äther bis zum Verschwinden der sauren Reaktion derWaschflüssigkeit mitWasser gewaschen. Die vereinigten wässerigen Flüssigkeiten wurden auf $70 \mathrm{~cm}^{3}$ eingekocht, in einem Meßkolben auf $100 \mathrm{~cm}^{3}$ aufgefüllt und in $5 \mathrm{~cm}^{3}$ dieser Flüssigkeit das Glyzerin nach dem Jodidverfahren bestimmt. Die ätherische Fett-Fettsäurelösung wurde in einen gewogenen Kolben gebracht, der Äther, zuletzt im luftverdünnten Raume, abdestilliert und schließlich das Gemisch bei $100^{\circ} \mathrm{C}$. im partiellen Wasserstoffvakuum zwei bis drei Stunden lang getrocknet. In einem aliquoten Teile des so behandelten FettFettsäuregemisches wurde die Säurezahl bestimmt. Aus der Zunahme dieser Säurezahl gegenüber der dem ursprünglichen Fette eigentümlichen ließ sich mit hinreichender Genauigkeit die in Reaktion getretene Kalimenge berechnen. Es ist ohneweiters klar, daß bei vollständig durchgeführter Verseifung für ein Molekül abgeschiedenes Glyzerin drei Moleküle Kali verbraucht werden, daß aber bei Unterbrechung des Prozesses das Verhältnis $1: 3$ bei Vorhandensein von Di- und 
Monoacylhydrinen nicht vorliegen kann. Die Versuche waren unter den für stufenweise Verseifung günstigsten Bedingungen angeordnet (Verwendung unzureichender Kalimengen und rechtzeitige Unterbrechung des Prozesses). Zur Verarbeitung gelangte ein käufliches Olivenöl, selbst ausgeschmolzener Talg, reines Tristearin und ein Präparat unter der Bezeichnung Trioleinn, das aber eine Säurezahl von 23 und eine Verseifungszahl von 200 besaß, gegenüber den theoretischen 0 und 190 . Den Versuch mit diesem Präparate halte ich infolgedessen auch nicht für konkludent.

Das zu den folgenden Versuchen verwendete Olivenöl besaß eine Säurezahl von $1 \cdot 9$ und eine Verseifungszahl von 190.9; der Glyzeringehalt (nach dem Jodidverfahren) betrug $10 \cdot 3 \%$.

I. $19 \cdot 133 \mathrm{~g} \ddot{\mathrm{O}} \mathrm{l}$ wurden mit $25 \mathrm{~cm}^{3} \mathrm{n}_{1} \mathrm{KOH}$ versetzt, wie oben beschrieben 6 Stunden erwärmt, zersetzt und ausgeschüttelt. $5 \mathrm{~cm}^{3}$ des Glyzerinwassers lieferten $0.0857 \mathrm{~g} \mathrm{AgJ}=0.0336 \mathrm{~g}$ Glyzeyin, einer Gesamtmenge von $0.672 \mathrm{~g}$ Glyzerin entsprechend. Das drei Stunden lang getrocknete Fett-Fettsäuregemisch wog $18 \cdot 7817 \mathrm{~g}$ und besaß eine Säurezahl von $66 \cdot 7$. Daraus berechnet sich ein Kaliverbrauch von $1.2185 \mathrm{~g}$. Bei quadrimolekularer Verseifung würde diesem entsprechen:

$$
\begin{array}{lll}
\text { Glyzerin ............ } & 0.666 \mathrm{~g} \text {, } \\
\text { gefunden Glyzerin .... } & 0.672 \mathrm{~g} \text {. }
\end{array}
$$

Dieser Versuch wurde noch weiter ausgeführt. Wenin auch sehr unwahrscheinlich, war doch die Möglichkeit vorhanden, daß eines der eventuell gebildeten Zwischenprodukte in Wasser löslicher sei als in Äther. Es hätte sich dann in dem mit Äther ausgeschüttelten Glyzerinwasser befinden müssen. Die von der Glyzerinbestimmung übriggebliebenen zirka $95 \mathrm{~cm}^{3}$ wurden mit einem Überschuß von $n / 1$ Kalilauge längere Zeit gekocht und dann mit Essigsäure angesäuert. Bei Anwesenheit nachweisbarer Mengen eines $Z$ wischenproduktes hätte eine Trübung von ausgeschiedener Fettsäure auftreten müssen. Die Flüssigkeit blieb aber vollständig klar. Der restliche Teil des Fett-Fettsäuregemisches wurde in Äther gelöst, die der bereits ermittelten Säurezahl entsprechende Menge von Kalilauge zugesetzt und dann durch Zusatz einer genügenden Menge Calciumacetat die Kaliseife in Calciumseife umgewandelt. Es trat zwar Emulsionsbildung ein, doch schied sich dieselbe nach mehrtägigem 
Stehen gut ab. Die Ätherfettlösung, in der die Calciumseife suspendiert war, ließ sich auf diese Weise vollkommen auswaschen. Schließlich wurde von der Seife abfiltriert, der Äther abdestilliert und im zurückbleibenden Fett Säurezahl, Verseifungszahl und Glyzerin bestimmt. Es wurde gefunden: S. Z. $2 \cdot 0$, V. Z. $190 \cdot 0$, Glyzerin $10 \cdot 21$. Die Übereinstimmung dieser Zahlen mit den im ursprünglichen Öl gefundenen, und zwar: S. Z. $1 \cdot 9$, V. Z. $190 \cdot 9$, Glyzerin $10 \cdot 3$, beweisen, daß in dem nach Entfernung des verseiften Ölanteiles zurückbleibenden Fett keine auf stattgehabte stufenweise Verseifung hindeutende Anteile sich befanden. Endlich wurde noch die Calciumseife nach dem Waschen und Trocknen der Behandlung mit Jodwasserstoffsäure unterzogen; wie erwartet lieferte dieselbe keine Spur von Jodsilber, war also frei von glyzerinhältigen Zwischenprodukten.

II. $30.576 \mathrm{~g}$ Ö1 wurden mit $25 \mathrm{~cm}^{3} n / 1 \mathrm{KOH}$ drei Stunden lang wie oben erhitzt und weiter behandelt. $5 \mathrm{~cm}^{3}$ des Glyzerinwassers lieferten $0.077 \mathrm{~g} \mathrm{AgJ}=0.0302 \mathrm{~g}$ Glyzerin, einer Gesamtmenge von $0.604 \mathrm{~g}$ Glyzerin entsprechend. Das Fett-Fettsäuregemisch wurde drei Stunden lang getrocknet, wog $30 \cdot 1643 \mathrm{~g}$ und besah eine Säurezahl von $37 \cdot 2$. Daraus berechnet sich ein Kaliverbranch von $1.0648 \mathrm{~g}$. Bei quadrimolekularer Verseifung würdc diesem entsprechen:

$$
\begin{aligned}
& \text { Glyzerin } \ldots \ldots \ldots \ldots \ldots .0 .582 g \\
& \text { gefunden Glyzerin....... } 0.604 \mathrm{~g} \text {. }
\end{aligned}
$$

III. $22 \cdot 674 \mathrm{~g}$ Rindstalg, der eine Säurezahl von $1 \cdot 1$ besaß, wurde mit $25 \mathrm{~cm}^{3} \mathrm{n} / \mathrm{KOH}$ drei Stunden lang wie oben erhitzt und dann weiter verarbeitet. $5 \mathrm{~cm}^{3}$ des Glyzerinwassers lieferten $0.0512 \mathrm{~g}$ AgJ $=0.02125 \mathrm{~g}$ Glyzerin, einer Gesamtmenge von $0.425 \mathrm{~g}$ Glyzerin entsprechend. Das FettFettsäuregemisch wurde zwei Stunden lang getrocknet, wog $22.378 \mathrm{~g}$ und besaß eine Säurezahl von $36 \cdot 1$. Daraus berechnet sich ein Verbrauch von $0.7832 \mathrm{~g}$ Kali. Bei quadrimolekularer Verseifung würde diesem entsprechen:

$$
\begin{array}{ll}
\text { Glyzerin } \ldots \ldots \ldots \ldots \ldots & 0.428 \mathrm{~g} \\
\text { gefunden Glyzerin. } \ldots \ldots \ldots & 0.425 \mathrm{~g}
\end{array}
$$

IV. Zum Versuche wurde Tristearin ${ }^{1}$ verwendet. Gewogen wurden $8.4475 \mathrm{~g}$; mit $15 \mathrm{~cm}^{3} n / \mathrm{KOH}$ drei Stunden lang wie oben erhitzt, mit Essigsäure zersetzt und, wegen der verhältnismäBig geringen Löslichlseit der

1 Die Substanz zeigte einen Schmelzpunkt von 70 bis $71^{\circ}$. Einmaliges Umiristallisieren aus heißem Benzol veränderte denselben nicht. Trotzdem besaß sie S. Z. von 1.8 und V. Z. $191 \cdot 6$ gegenüber den von der Theorie geforderten 0 und 189. 
Stearinsäure in Äther, mit heißem Benzol ausgeschüttelt. Der wässerige Antei1 wurde mit heißem Benzol, die Benzollösung mit heißem Wasser wieder bis zum Verschwinden der sauren Reaktion in der letzten Waschflüssigkeit gewaschen. $5 \mathrm{~cm}^{3}$ des Glyzerinwassers lieferten $0.0415 \mathrm{~g} \mathrm{AgJ}=0.01627 \mathrm{~g}$ Glyzerin, einer Gesamtmenge von $0.3254 \mathrm{~g}$ Glyzerin entsprechend. Das FettFettsäuregemisch, zwei Stunden lang getrocknet, wog $8.2587 \mathrm{~g}$ und besaß eine Säurezahl von 74.7. Daraus berechnet sich ein Kaliverbrauch von $0.602 g$. Bei quadrimolekularer Verseifung würde diesem entsprechen :

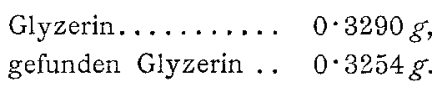

Sehließlich sei noch erwähnt, daß beim Versuche mit Triolein, den ich wegen der unreinen Beschaffenheit der Substanz nicht für beweisend ansehe, bei ganz gleicher Behandlungsweise ein Verlust von $3.5 \%$ Glyzerin gegenüber der dem Kaliverbrauch entsprechenden Menge resultierte.

Bei Betrachtung der Versuchsresultate fällt sofort auf, daß bei den mit Olivenöl angestellten Versuchen mehr Glyzerin gefunden wurde, als der verbrauchten Kalimenge entspricht. Da nicht anzunehmen ist, daß sich bei $100^{\circ} \mathrm{C}$. das Wasser verseifend an der Reaktion beteiligt, mußte dieser Umstand eine andere Ursache haben. Bei Versuch II, bei dem das Plus den Betrag von $3 \%$ noch übersteigt, wurde bei verhältnismäßig sehr gutem und konstantem Vakuum $(22 \mathrm{~mm}$, während dasselbe bei Versuch I zwischen 40 und $60 \mathrm{~mm}$ schwankte) drei Stunden lang bei $100^{\circ} \mathrm{C}$. getrocknet. Ein zu Anfang der dritten Stunde in das Kühlerrohr eingelegtes angefeuchtetes blaues Lackmuspapier färbte sich in ganz kurzer Zeit deutlich rot; da das Fett-Fettsäuregemisch solange gewaschen worden war, bis das Waschwasser nicht mehr sauer reagierte, also die Essigsäure schon zu Beginn der Trocknung nicht mehr vorhanden war, eventuelle Spuren aber jedenfalls während des zweistündigen Trocknens gänzlich entfernt worden waren, so ist diese Rötung nur dadurch zu erklären, daß bei dieser Temperatur und diesem niederen Druck sich bereits Anteile eines sauer reagierenden Stoffes verflüchtigen. Daraus ließe sich natürlich $z$ wanglos ein geringerer Kaliverbrauch und dementsprechend das Plus an Glyzerin erklären. Es hat den Anschein, als ob diese flüchtige Säure durch bei der Verarbeitung auch unter so schonenden Bedingungen nicht ganz zu verhindernde Oxydation gebildet wird. Beim Talg, der nur 
wenig, und beim Tristearin, das durch Oxydation gar nicht angegriffen wird, ist ein Plus an Glyzerin auch nicht aufgetreten, sondern im Gegenteil ein kleines, durch normale Arbeitsverluste bedingtes Minus.

Wenn nun auch bei den Versuchen mit Olivenöl die Differenzen im Glyzerinkaliverhältnis nicht groß sind und gerade in entgegengesetzter Richtung liegen, wie wenn die Verseifung stufenweise erfolgt wäre, haben dieselben durch den erwähnten Umstand doch etwas an Beweiskraft eingebüßt. Immerhin berechtigten mich aber die Erfahrungen, die ich bei Versuch I gemacht habe, dann die Resultate der Versuche mit Talg und Tristearin, die mit möglichster Exaktheit ausgeführt wurden - immer nur bezüglich Verseifung von Fett mit Kali in inhomogener Lösung - zu den Behauptungen:

1. Das Vorhandensein von Di- und Monoacylhydrinen in partiell verseiften Fetten ist nicht nachweisbar.

2. Die Verseifung von Fetten durch Kali in inhomogener Lösung verläuft praktisch quadrimolekular. 